\title{
Total Heat Integration in the Biobutanol Separation Process
}

\section{Electronic Supporting Information}

Ramón González-Bravo, ${ }^{\mathrm{a}}$ Eduardo Sánchez-Ramírez, Juan José Quiroz-Ramírez, Juan Gabriel SegoviaHernández, ${ }^{\mathrm{b}}$ Luis Fernando Lira-Barragán, ${ }^{\mathrm{a}}$ José María Ponce-Ortega ${ }^{a^{*}}$

${ }^{a}$ Chemical Engineering Department, Universidad Michoacana de San Nicolás de Hidalgo, Morelia, Michoacán, 58060, México

${ }^{b}$ Chemical Engineering Department, Universidad de Guanajuato, Guanajuato, Guanajuato, 36050, México 
This supporing information containts additional details for the designs identified in the paper. 
Table 1. Feed characterization.

\begin{tabular}{c|c}
\hline Parameter & Value \\
\hline Temperature $(\mathrm{K})$ & 322 \\
Vapor fraction & 0 \\
Flowrate $\left(\mathrm{kg} \mathrm{h}^{-1}\right)$ & 45,3592 \\
Composition $(\mathrm{mol} \mathrm{\% )}$ & \\
\hline Biobutanol & 0,1128 \\
Acetone & 0,0808 \\
\hline Ethanol & 0,0043 \\
Water & 0,80198 \\
\hline
\end{tabular}

Table 2. Design parameters for the reference configuration of Figure 3 (3C).

\begin{tabular}{c|c|c|c|c}
\hline Parameter & Extractor & $\mathrm{C} 1$ & $\mathrm{C} 2$ & $\mathrm{C} 3$ \\
\hline Number of theoretical stages & 5 & 26 & 46 & 20 \\
Reflux ratio & --- & 0.905 & 6.034 & 14.836 \\
Feed stage & 1 & 13 & 32 & $5 / 15$ \\
\hline Solvent feeed stage & 5 & --- & --- & --- \\
Side stream stage & --- & --- & 44 & --- \\
Column diameter $(\mathrm{m})$ & 0.335 & 0.322 & 0.325 & 0.292 \\
Operative pressure $(\mathrm{kPa})$ & 101.353 & 101.353 & 101.353 & 101.353 \\
\hline Distillate flowrate $\left(\mathrm{kg} \mathrm{h}^{-1}\right)$ & --- & 21.687 & 7.694 & 0.333 \\
\hline Side stream flowrate $\left(\mathrm{kg} \mathrm{h}^{-1}\right)$ & --- & --- & 1.901 & --- \\
\hline Solvent flowrate $\left(\mathrm{kg} \mathrm{h}^{-1}\right)$ & 708.549 & -- & --- & -- \\
\hline Solvent makeup $\left(\mathrm{kg} \mathrm{h}^{-1}\right)$ & 0.709 & --- & --- & --- \\
\hline Condenser duty $\left(\mathrm{kW}^{-1}\right)$ & --- & 7.284 & 7.736 & 1.239 \\
\hline Reboiler duty $(\mathrm{kW})$ & --- & 65.919 & 8.428 & 0.907 \\
\hline
\end{tabular}


Table 3. Design parameters for the thermally coupled configuration of Figure 4 (4B).

\begin{tabular}{c|c|c|c|c}
\hline Parameter & Extractor & $\mathrm{C} 1$ & $\mathrm{C} 2$ & $\mathrm{C} 3$ \\
\hline Number of theoretical stages & 5 & 26 & 46 & 20 \\
\hline Reflux ratio & -- & --- & 5.424 & 14.631 \\
\hline Feed stage & 1 & 13 & 32 & $5 / 15$ \\
\hline Solvent feed stage & 5 & -- & - & --- \\
\hline Side stream stage & -- & -- & 44 & --- \\
\hline Column diameter $(\mathrm{m})$ & 0.335 & 0.321 & 0.325 & 0.287 \\
\hline Operative pressure $(\mathrm{kPa})$ & 101.353 & 101.353 & 101.353 & 101.353 \\
\hline Distillate flowrate $\left(\mathrm{kg} \mathrm{h}^{-1}\right)$ & --- & -- & 7.716 & 0.332 \\
\hline Thermal coupling flowrate $\left(\mathrm{kg} \mathrm{h}^{-1}\right)$ & --- & --- & 14.978 & 9.139 \\
\hline Side stream flowrate $\left(\mathrm{kg} \mathrm{h}^{-1}\right)$ & -- & --- & 2.900 & --- \\
\hline Solvent flowrate $\left(\mathrm{kg} \mathrm{h}^{-1}\right)$ & 708.465 & --- & --- & --- \\
\hline Solvent makeup $\left(\mathrm{kg} \mathrm{h}^{-1}\right)$ & 0.709 & --- & --- & --- \\
\hline Condenser duty $\left(\mathrm{kW}^{-1}\right)$ & --- & 0.000 & 7.096 & 1.214 \\
\hline Reboiler duty $\left(\mathrm{kW}^{-1}\right)$ & --- & 65.082 & 0.000 & 2.222 \\
\hline
\end{tabular}

Table 4. Design parameters for the intensified design of Figure 5 (5D).

\begin{tabular}{c|c|c|c}
\hline Parameter & Extractor & $\mathrm{C} 1$ & $\mathrm{C} 2$ \\
\hline Number of theoretical stages & 5 & 58 & 20 \\
\hline Reflux ratio & -- & 27.182 & --- \\
\hline Feed stage & 1 & 45 & --- \\
\hline Solvent feed stage & 5 & --- & --- \\
Side stream stage & -- & -- & 12 \\
\hline Column diameter $(\mathrm{m})$ & 0.335 & 0.323 & 0.324 \\
\hline Operative pressure $(\mathrm{kPa})$ & 101.353 & 101.353 & 101.353 \\
\hline Distillate flowrate $\left(\mathrm{kg} \mathrm{h}^{-1}\right)$ & --- & 7.711 & --- \\
\hline Thermal coupling flowrate $\left(\mathrm{kg} \mathrm{h}^{-1}\right)$ & -- & 118.621 & --- \\
\hline Side stream flowrate $\left(\mathrm{kg} \mathrm{h}^{-1}\right)$ & -- & --- & 0.336 \\
\hline Solvent flowrate $\left(\mathrm{kg} \mathrm{h}^{-1}\right)$ & 708.289 & --- & --- \\
\hline Solvent makeup $\left(\mathrm{kg} \mathrm{h}^{-1}\right)$ & 0.684 & --- & --- \\
\hline Condenser duty $\left(\mathrm{kW}^{-1}\right)$ & --- & 31.094 & 0.000 \\
\hline Reboiler duty $\left(\mathrm{kW}^{-1}\right.$ & --- & 65.642 & 24.517 \\
\hline
\end{tabular}


Table 5. Data for the biobutanol separation configuration.

\begin{tabular}{|c|c|c|c|c|c|c|c|}
\hline CASE & & STREAM & No. & TIN (K) & TOUT (K) & FCP $(\mathrm{kW} / \mathrm{K})$ & $\mathrm{Q}(\mathrm{kW})$ \\
\hline \multirow{13}{*}{$3 \mathrm{~A}$} & \multirow{8}{*}{ HS } & $\mathrm{VCI}$ & 1 & 361.98 & 361.98 & 25.39 & -25.39 \\
\hline & & VCII & 2 & 329.79 & 329.79 & 28.26 & -28.26 \\
\hline & & VCIII & 3 & 329.3 & 329.3 & 2.116 & -21.16 \\
\hline & & TOP C & 4 & 329.3 & 303.15 & 0.003215 & -0.0841 \\
\hline & & BOTTOM W & 5 & 373.18 & 303.15 & 0.007893 & -0.553 \\
\hline & & BOTTOM C & 6 & 351.35 & 303.15 & 0.0002405 & -0.0116 \\
\hline & & BOTTOM D & 7 & 373.18 & 303.15 & 0.006515 & -0.456 \\
\hline & & BOTTOM E & 8 & 388.88 & 303.15 & 0.01273 & -1.09 \\
\hline & \multirow{5}{*}{$\mathrm{CS}$} & VRI & 1 & 373.1 & 373.1 & 27.33 & 27.33 \\
\hline & & VRII & 2 & 365.68 & 365.68 & 28.61 & 28.61 \\
\hline & & VRIII & 3 & 351.35 & 351.35 & 21.15 & 21.15 \\
\hline & & VRIV & 4 & 373.18 & 373.18 & 5.79 & 5.79 \\
\hline & & VRV & 5 & 383.54 & 383.54 & 5.49 & 5.49 \\
\hline \multirow{14}{*}{ 3B } & \multirow{10}{*}{ HS } & VCI & 1 & 361.96 & 361.96 & 25.1 & -25.1 \\
\hline & & VCII & 2 & 329.32 & 329.32 & 15.55 & -15.55 \\
\hline & & VCIII & 3 & 358.87 & 358.87 & 2.116 & -21.16 \\
\hline & & VCIV & 4 & 358.87 & 358.87 & 0.2005 & -2.01 \\
\hline & & BOTTOM W & 5 & 373.18 & 303.15 & 0.001885 & -0.132 \\
\hline & & TOP B & 6 & 329.31 & 303.15 & 0.0007732 & -0.0202 \\
\hline & & WASTE A & 7 & 365.18 & 303.15 & 0.009921 & -0.615 \\
\hline & & TOP C & 8 & 355.43 & 303.15 & 0.0002402 & -0.0126 \\
\hline & & TOP D & 9 & 365.01 & 303.15 & 0.007008 & -0.434 \\
\hline & & BOTTOM D & 10 & 390.84 & 303.15 & 0.008583 & -0.753 \\
\hline & \multirow{4}{*}{$\mathrm{CS}$} & VRI & 1 & 373.18 & 373.18 & 28.59 & 28.59 \\
\hline & & VRII & 2 & 364.73 & 364.73 & 15.78 & 15.78 \\
\hline & & VRIII & 3 & 365.31 & 365.31 & 21.15 & 21.15 \\
\hline & & VRIV & 4 & 365.31 & 365.31 & 2.01 & 2.01 \\
\hline \multirow{10}{*}{$3 \mathrm{C}$} & \multirow{7}{*}{$\mathrm{HS}$} & $\mathrm{VCI}$ & 1 & 377.34 & 377.34 & 7.28 & -7.28 \\
\hline & & VCII & 2 & 329.3 & 329.3 & 7.74 & -7.74 \\
\hline & & VCIII & 3 & 351.06 & 351.06 & 0.1 & -1 \\
\hline & & TOP B & 4 & 329.3 & 303.15 & 0.003687 & -0.1 \\
\hline & & TOP C & 5 & 349.97 & 303.15 & 0.000181 & -0.00848 \\
\hline & & BOTTOM C & 6 & 390.79 & 303.15 & 0.008203 & -0.719 \\
\hline & & SEPARAB & 7 & 444.35 & 303.15 & 0.4166 & -58.82 \\
\hline & \multirow{3}{*}{$\mathrm{CS}$} & VRI & 1 & 444.32 & 444.32 & 65.92 & 65.92 \\
\hline & & VRII & 2 & 389.06 & 389.06 & 8.43 & 8.43 \\
\hline & & VRIII & 3 & 390.69 & 390.69 & 0.91 & 0.91 \\
\hline $3 \mathrm{D}$ & HS & VCII & 1 & 329.31 & 329.31 & 11.84 & -11.84 \\
\hline
\end{tabular}




\begin{tabular}{|c|c|c|c|c|c|c|c|}
\hline & & VCIII & 2 & 351.16 & 351.16 & 1.28 & -1.28 \\
\hline & & TOP B & 3 & 329.31 & 303.15 & 0.00322 & -0.0843 \\
\hline & & TOP C & 4 & 350.21 & 303.15 & 0.000269 & -0.0126 \\
\hline & & BOTTOM C & 5 & 390.87 & 303.15 & 0.0127 & -1.12 \\
\hline & & SEPARAB & 6 & 444.34 & 303.15 & 0.468 & -66.12 \\
\hline & \multirow{3}{*}{$\mathrm{CS}$} & VRI & 1 & 444.22 & 161.07 & 65.92 & 65.92 \\
\hline & & VRII & 2 & 390.93 & 107.78 & 0.55 & 5.55 \\
\hline & & VRIII & 3 & 444.22 & 161.07 & 1.13 & 11.26 \\
\hline \multirow{9}{*}{$4 \mathrm{~A}$} & \multirow{7}{*}{ HS } & $\mathrm{VCI}$ & 1 & 377.34 & 377.34 & 7.28 & -7.28 \\
\hline & & VCII & 2 & 329.3 & 329.3 & 9.62 & -9.62 \\
\hline & & VCIII & 3 & 350.82 & 350.82 & 0.128 & -1.28 \\
\hline & & TOP B & 4 & 329.3 & 303.15 & 0.003217 & -0.0841 \\
\hline & & TOP C & 5 & 349.63 & 303.15 & 0.0002787 & -0.013 \\
\hline & & BOTTOM C & 6 & 390.85 & 303.15 & 0.01243 & -1.09 \\
\hline & & SEPARAB & 7 & 444.35 & 303.15 & 0.4183 & -59.06 \\
\hline & \multirow{2}{*}{$\mathrm{CS}$} & VRI & 1 & 444.32 & 444.32 & 65.92 & 65.92 \\
\hline & & VRIII & 2 & 390.79 & 390.79 & 11.26 & 11.26 \\
\hline \multirow{8}{*}{$4 \mathrm{~B}$} & \multirow{6}{*}{ HS } & VCII & 1 & 329.32 & 329.32 & 7.1 & -7.1 \\
\hline & & VCIII & 2 & 351.16 & 351.16 & 1.22 & -1.22 \\
\hline & & TOP B & 3 & 329.31 & 303.15 & 0.00323 & -0.0845 \\
\hline & & TOP C & 4 & 351.85 & 303.15 & 0.0002723 & -0.0133 \\
\hline & & BOTTOM C & 5 & 390.9 & 303.15 & 0.00763 & -0.67 \\
\hline & & SEPARAB & 6 & 444.35 & 303.15 & 0.4397 & -62.08 \\
\hline & \multirow{2}{*}{$\mathrm{CS}$} & VRI & 1 & 444.31 & 444.31 & 65.08 & 65.08 \\
\hline & & VRIII & 2 & 390.79 & 390.79 & 2.22 & 2.22 \\
\hline \multirow{8}{*}{$4 \mathrm{C}$} & \multirow{6}{*}{ HS } & $\mathrm{VCI}$ & 1 & 329.3 & 329.3 & 8.05 & -8.05 \\
\hline & & VCIII & 2 & 353.46 & 353.46 & 1.27 & -1.27 \\
\hline & & TOP A & 3 & 329.3 & 303.15 & 0.00323 & -0.0843 \\
\hline & & TOP C & 4 & 351.87 & 303.15 & 0.00028 & -0.0136 \\
\hline & & BOTTOM C & 5 & 390.9 & 303.15 & 0.01193 & -1.05 \\
\hline & & SEPARAB & 6 & 444.36 & 303.15 & 0.3847 & -54.32 \\
\hline & \multirow{2}{*}{$\mathrm{CS}$} & VRI & 1 & 444.36 & 444.36 & 65.75 & 65.75 \\
\hline & & VRIII & 2 & 390.86 & 390.86 & 2.56 & 2.56 \\
\hline \multirow{8}{*}{$4 \mathrm{D}$} & \multirow{6}{*}{ HS } & VCII & 1 & 329.31 & 329.31 & 7.48 & -7.48 \\
\hline & & VCIII & 2 & 352.67 & 352.67 & 0.43 & -0.43 \\
\hline & & TOP B & 3 & 329.3 & 303.15 & 0.00322 & -0.0843 \\
\hline & & TOP C & 4 & 351.21 & 303.15 & 0.0002721 & -0.0131 \\
\hline & & BOTTOMB & 5 & 390.81 & 303.15 & 0.01262 & -1.11 \\
\hline & & SEPARAB & 6 & 444.34 & 303.15 & 0.4676 & -66.03 \\
\hline & \multirow{2}{*}{$\mathrm{CS}$} & VRI & 1 & 444.23 & 444.23 & 65.19 & 65.19 \\
\hline & & VRII & 2 & 390.64 & 390.64 & 1.71 & 1.71 \\
\hline
\end{tabular}




\begin{tabular}{|c|c|c|c|c|c|c|c|}
\hline \multirow{8}{*}{$5 \mathrm{~A}$} & \multirow{6}{*}{ HS } & $\mathrm{VCI}$ & 1 & 329.34 & 329.34 & 8.05 & -8.05 \\
\hline & & VCIII & 2 & 353.26 & 353.26 & 1.27 & -1.27 \\
\hline & & TOP A & 3 & 329.33 & 303.15 & 0.00362 & -0.0948 \\
\hline & & TOP C & 4 & 351.89 & 303.15 & 0.0002592 & -0.0126 \\
\hline & & BOTTOMB & 5 & 390.92 & 303.15 & 0.01097 & -0.963 \\
\hline & & SEPARAB & 6 & 444.36 & 303.15 & 0.3847 & -54.32 \\
\hline & \multirow{2}{*}{$\mathrm{CS}$} & VRI & 1 & 444.36 & 444.36 & 65.35 & 65.35 \\
\hline & & VRII & 2 & 390.9 & 390.9 & 3.41 & 3.41 \\
\hline \multirow{8}{*}{$5 \mathrm{~B}$} & \multirow{6}{*}{ HS } & $\mathrm{VCI}$ & 1 & 329.33 & 329.33 & 54.59 & -54.59 \\
\hline & & VCII & 2 & 351.55 & 351.55 & 0.22 & -0.22 \\
\hline & & TOP A & 3 & 329.32 & 303.15 & 0.00324 & -0.0848 \\
\hline & & TOP B & 4 & 349.97 & 303.15 & 0.000267 & -0.0125 \\
\hline & & BOTTOMB & 5 & 390.88 & 303.15 & 0.01236 & -1.08 \\
\hline & & SEPARAB & 6 & 444.36 & 303.15 & 0.3847 & -54.32 \\
\hline & \multirow{2}{*}{$\mathrm{CS}$} & VRI & 1 & 444.36 & 444.36 & 100.95 & 100.95 \\
\hline & & VRII & 2 & 390.77 & 390.77 & 12.85 & 12.85 \\
\hline \multirow{7}{*}{$5 \mathrm{C}$} & \multirow{5}{*}{ HS } & VCII & 1 & 351.55 & 351.55 & 37.15 & -37.15 \\
\hline & & TOP B & 2 & 329.3 & 303.15 & 0.0322 & -0.0843 \\
\hline & & SSB & 3 & 353.53 & 303.15 & 0.00016 & -0.00808 \\
\hline & & BOTTOMB & 4 & 390.67 & 303.15 & 0.01268 & -1.11 \\
\hline & & SEPARAB & 5 & 444.34 & 303.15 & 0.4634 & -65.43 \\
\hline & \multirow{2}{*}{$\mathrm{CS}$} & VRI & 1 & 444.26 & 444.26 & 65.67 & 65.67 \\
\hline & & VRII & 2 & 389.93 & 389.93 & 30.54 & 30.54 \\
\hline \multirow{7}{*}{$5 \mathrm{D}$} & \multirow{5}{*}{ HS } & $\mathrm{VCI}$ & 1 & 329.31 & 329.31 & 3.11 & -3.11 \\
\hline & & TOP A & 2 & 329.3 & 303.15 & 0.0323 & -0.0844 \\
\hline & & SSB & 3 & 354.02 & 303.15 & 0.000168 & -0.00853 \\
\hline & & BOTTOMB & 4 & 390.85 & 303.15 & 0.01254 & -1.1 \\
\hline & & SEPARAB & 5 & 444.36 & 303.15 & 0.3847 & -54.32 \\
\hline & \multirow{2}{*}{$\mathrm{CS}$} & VRI & 1 & 444.36 & 444.36 & 65.64 & 65.64 \\
\hline & & VRII & 2 & 390.64 & 390.64 & 24.52 & 24.52 \\
\hline
\end{tabular}


Table 6. Economic results.

\begin{tabular}{|c|c|c|c|c|c|c|c|c|c|c|c|c|}
\hline Case & TPS (\$/y) & $\begin{array}{l}\text { TCR } \\
(\$ / y)\end{array}$ & $\begin{array}{l}\mathrm{CC} \\
(\$ / \mathrm{y})\end{array}$ & $\mathrm{FC}(\$ / \mathrm{y})$ & $\mathrm{OC}(\$ / \mathrm{y})$ & $\begin{array}{l}\mathrm{ESC} \\
(\$ / y)\end{array}$ & $\begin{array}{l}\text { CCSS } \\
(\$ / y)\end{array}$ & $\begin{array}{l}\text { TAC } \\
(\$ / y)\end{array}$ & $\begin{array}{l}\mathrm{BS} \\
(\$ / \mathrm{y})\end{array}$ & $\begin{array}{c}\text { OGGE } \\
\text { (ton } \mathrm{CO}_{2} \mathrm{eq} / \mathrm{y} \text { ) }\end{array}$ & NJG & TAP $(\$ / y)$ \\
\hline $3 \mathrm{~A}$ & $1,521,949$ & 10,330 & 814 & 54,418 & 40,851 & 460,490 & 400,861 & 957,434 & 159,778 & 62,739 & 705 & 734,623 \\
\hline $3 \mathrm{~B}$ & $1,521,010$ & 10,330 & 642 & 58,604 & 41,017 & 459,493 & 471,446 & $1,031,202$ & 160,870 & 62,597 & 705 & 661,008 \\
\hline $3 \mathrm{C}$ & $1,521,570$ & 10,330 & 740 & 41,860 & 40,918 & 460,084 & 94,434 & 638,036 & 162,125 & 62,681 & 705 & $1,055,989$ \\
\hline $3 \mathrm{D}$ & $1,521,930$ & 10,330 & 809 & 37,674 & 40,854 & 460,470 & 84,787 & 624,594 & 162,888 & 62,736 & 705 & $1,070,554$ \\
\hline $4 \mathrm{~A}$ & $1,521,720$ & 10,330 & 766 & 37,674 & 40,892 & 460,242 & 76,466 & 616,040 & 161,740 & 62,704 & 705 & $1,077,750$ \\
\hline $4 \mathrm{~B}$ & $1,521,200$ & 10,330 & 678 & 33,488 & 40,983 & 459,695 & 74,038 & 608,882 & 163,884 & 62,626 & 705 & $1,086,532$ \\
\hline $4 \mathrm{C}$ & $1,521,000$ & 10,330 & 658 & 33,488 & 41,020 & 459,476 & 73,406 & 608,048 & 163,392 & 62,595 & 705 & $1,086,674$ \\
\hline $4 \mathrm{D}$ & $1,521,340$ & 10,330 & 694 & 33,488 & 40,959 & 459,838 & 73,051 & 608,030 & 163,973 & 62,646 & 705 & $1,087,613$ \\
\hline $5 \mathrm{~A}$ & $1,521,010$ & 10,330 & 660 & 33,488 & 41,018 & 459,486 & 73,375 & 608,027 & 163,561 & 62,596 & 705 & $1,086,874$ \\
\hline $5 \mathrm{~B}$ & $1,523,800$ & 10,330 & 1084 & 33,488 & 40,523 & 462,462 & 69,987 & 607,544 & 163,226 & 63,019 & 705 & $1,089,812$ \\
\hline $5 \mathrm{C}$ & $1,523,120$ & 10,330 & 913 & 29,302 & 40,644 & 461,734 & 53,996 & 586,589 & 167,154 & 62,916 & 705 & $1,114,015$ \\
\hline $5 \mathrm{D}$ & $1,521,330$ & 10,330 & 676 & 29,302 & 40,961 & 459,829 & 57,509 & 588,277 & 168,849 & 62,645 & 705 & $1,112,232$ \\
\hline CCSS & $\begin{array}{r}\mathrm{TPS}=\mathrm{T} \\
\mathrm{TCR}=\mathrm{Ta} \\
\mathrm{CC}= \\
\text { Capital cos }\end{array}$ & $\begin{array}{l}\text { al power } \\
\text { credit red } \\
\text { Capital co } \\
\text { of the sep }\end{array}$ & $\begin{array}{l}\text { ales } \\
\text { ction } \\
\text { ation se }\end{array}$ & quence & & $\begin{array}{r}\mathrm{FC} \\
\mathrm{OC}=\mathrm{C} \\
\mathrm{SC}=\mathrm{E} \\
\mathrm{BS}=\end{array}$ & $\begin{array}{l}=\text { Fixed co } \\
\text { perational } \\
\text { ergy sour } \\
\text { Butanol S }\end{array}$ & costs & & \multicolumn{3}{|c|}{$\begin{array}{c}\mathrm{OGGE}=\text { Greenhouse gas emissions } \\
\mathrm{NJG}=\text { Number of jobs generated }\end{array}$} \\
\hline
\end{tabular}

